\title{
CORRESPONDENCE
}

\section{Experiences of using a single post-contrast CT scan of the urinary tract after triphasic contrast injection ${ }^{1}$}

To the Editor: In my article entitled 'Experiences of using a single postcontrast CT scan of the urinary tract after triphasic contrast injection' that was published in the SAJR Vol. 15, No. 4 (December 2011), I emphasised that we had not gained experience in this technique with renal pathologies such as pyelonephritis and small renal tumours. At a CT congress in South Africa last year, an international speaker and expert in CT also raised his concern about missing small renal tumours when questioned about this technique. I have had further experience since, and would like to share the following 2 cases, suggesting that this technique will demonstrate these 2 pathologies adequately.

\section{Case 1}

A 70-year-old man was diagnosed with a small right renal mass, using the classic post-contrast scan technique (arterial, nephrographic and

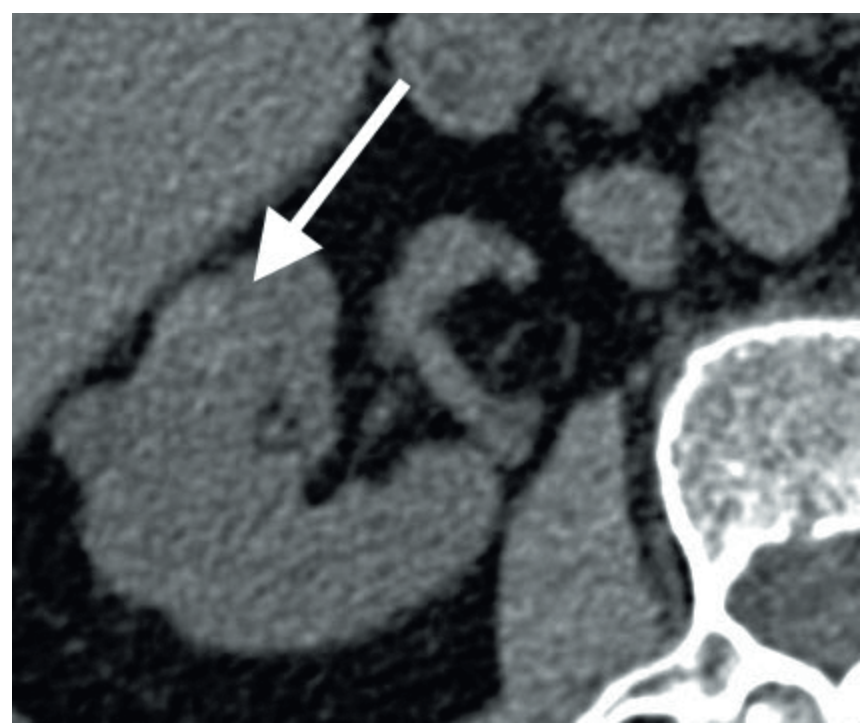

Fig. 1a. Pre contrast

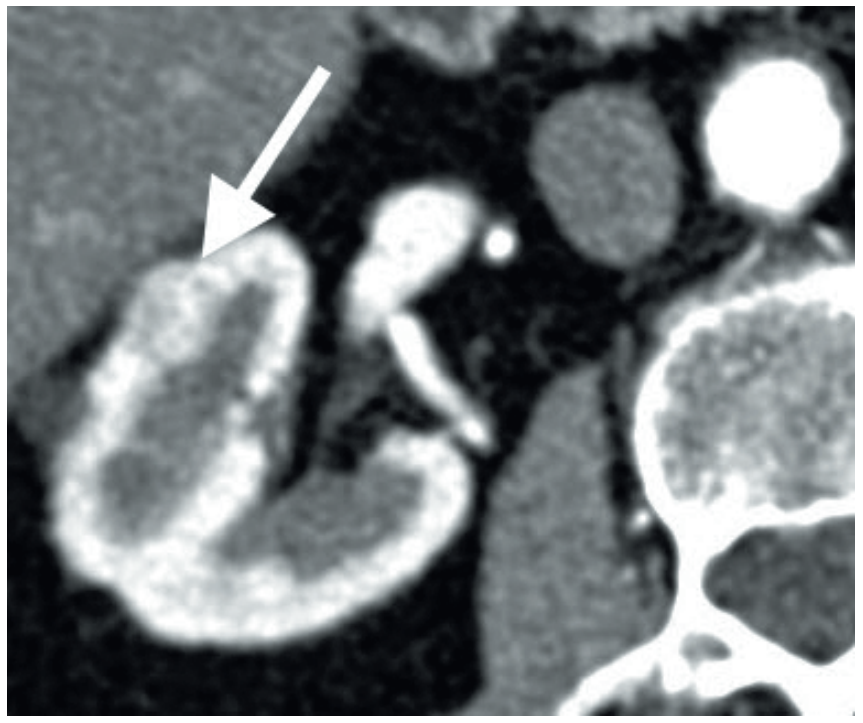

Fig. 1b. Arterial phase. delay excretory series). I performed a 6-month follow-up using the single post-contrast scan after a triphasic contrast injection.

Fig. $1(\mathrm{a}-\mathrm{d})$ shows the lesion identified at original scan and provisionally diagnosed as an oncocytoma. Fig. $2(\mathrm{a}-\mathrm{b})$ shows the lesion (arrow) at 6-month follow-up, unchanged. The lesion was a subtle lesion, difficult to identify on both examinations, but it is my opinion that it is seen as easily using the single post-triphasic contrast injection technique (Fig. 2) as on the classical 3 post-contrast series technique (Fig. 1).

\section{Case 2}

A 76-year-old woman presented with symptoms of urinary tract pathology. Pyelonephritis was diagnosed after a CT scan using the single-series post-triphasic contrast injection technique. We

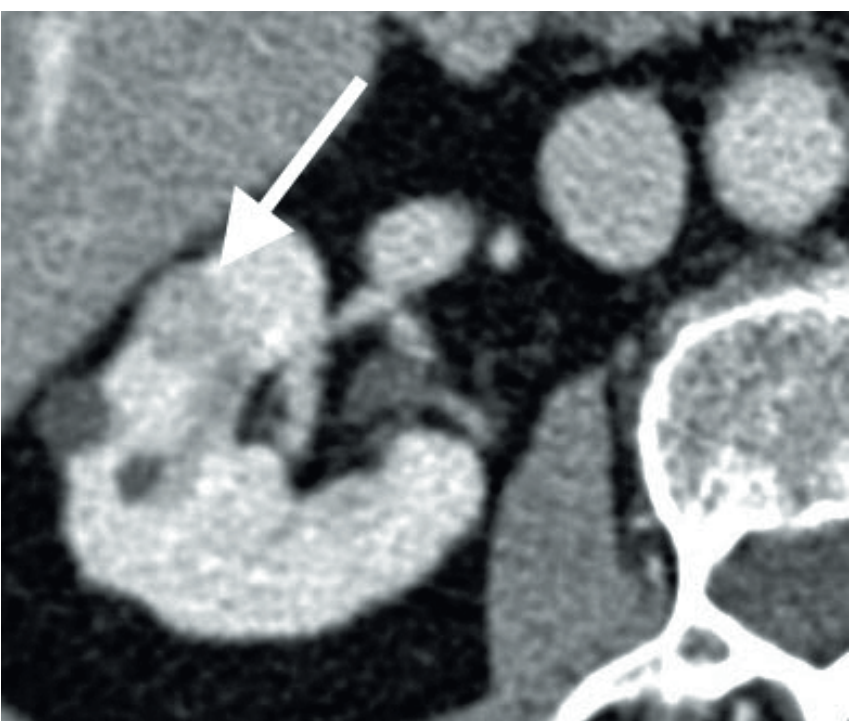

Fig. 1c. Nephrographic phase.

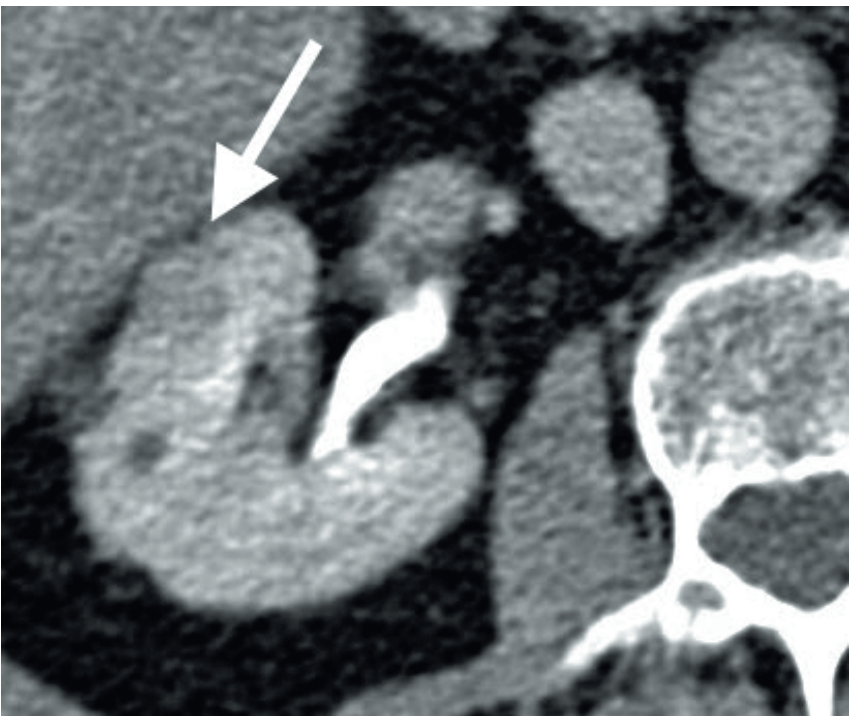

Fig. 1d. Delay excretory phase. 


\section{CORRESPONDENCE}

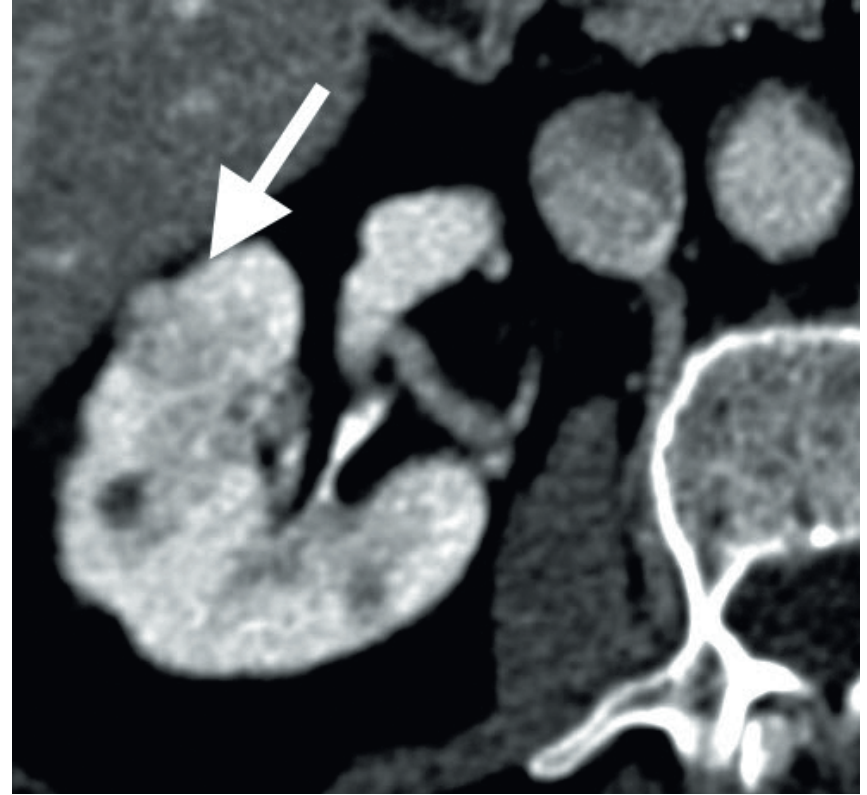

Fig. 2a. Axial view after single post-triphasic contrast injection series

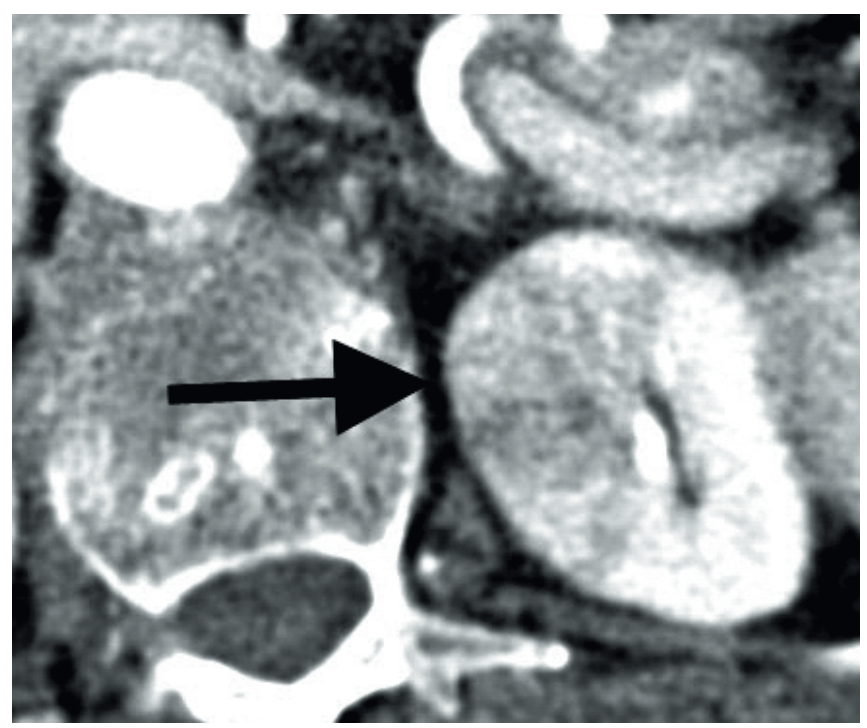

Fig. 3a. Axial left kidney.

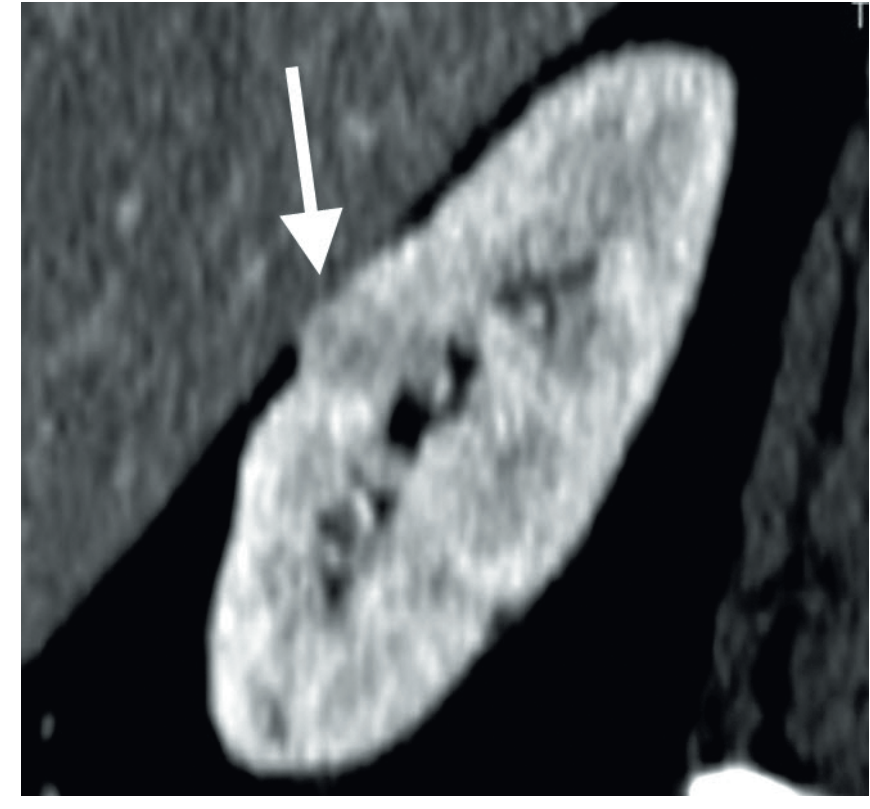

Fig. 2b. Sagittal reconstruction.

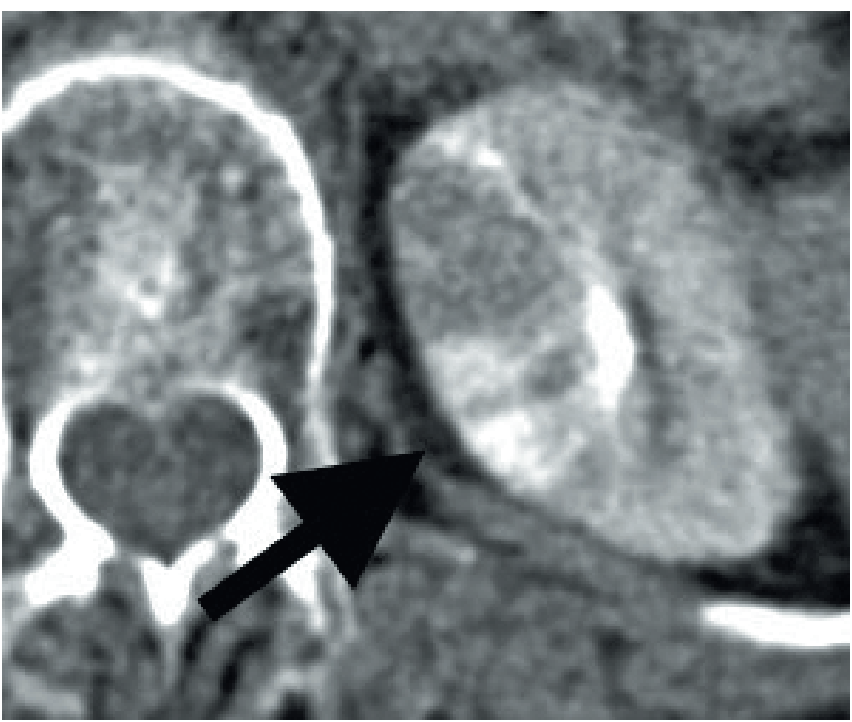

Fig. 3b. Axial left kidney after 3-hour post-contrast delay.

Fig. 3. The left kidney shows an area of inhomogenous poor contrast enhancement antero-medially on the initial scan (black arrow in Fig. 3a) after the triphasic contrast injection. On the 3-hour delay post-contrast scan, there is retention of contrast in the tubules, causing streaky contrast staining in the area of inflammation, typical of pyelonephritis (black arrow in Fig. 3b).

include a 3-hour post-contrast study on our patients if we suspect pyelonephritis.

These two cases show that this modified post-contrast technique is sensitive to small focal lesions and diffuse lesions within the kidneys. I acknowledge that a few cases do not constitute definitive scientific evidence that subtle lesions will not be missed; however, these types of case add to the radiologist's confidence in using this technique, bearing in mind that this technique replaces 3 post-contrast scans with a single scan and also effectively halves the overall radiation dose to the patient.

I thank my partners Dr Jaco Parsons for his initial diagnosis of the small renal mass (Case 1) and Dr Etienne Steenkamp for his case of pyelonephritis (Case 2).

\section{Phil Pretorius}

Drs Visser, Erasmus, Vawda \& Partners

Port Elizabeth

ppret@telkomsa.net

\footnotetext{
1. Pretorius, P. Experiences of using a single post-contrast CT scan of the urinary tract after triphasic contrast injection. South African Journal of Radiography 2011;15(4):140-145.
}

\section{S Afr J Rad 2012;16(3): 122-123. DOI:10.7196/SAJR.699}

